\title{
Tumor estromal gastrointestinal duodenal: relato de caso e importância da
}

\section{ecoendoscopia}

\author{
Duodenal gastrointestinal stromal tumor: case report and importance of ecoendoscopy \\ Tumor estromal duodenal gastrointestinal: reporte de caso e importancia de la ecoendoscopia
}

Recebido: 13/08/2021 | Revisado: 19/08/2021 | Aceito: 23/08/2021 | Publicado: 25/08/2021

Brian França dos Santos
ORCID: https://orcid.org/0000-0003-1574-630X
Universidade Iguaçu - LAGCAD-UNIG/RJ, Brasil
E-mail: drbrianfranca@hotmail.com
Vilson de Lemos Junior
ORCID: https://orcid.org/0000-0002-5478-2605
E-mail: vilson@hucff.ufrj.br
Juliana Ribeiro da Costa Lino
Centro Especializado em Doenças Digestivas e do Fígado - CEDIFI, Brasil
ORCID: https://orcid.org/0000-0002-9354-8694
E-mail: julianarclino@gmail.com
Cleber Queiroz Leite
ORCID: https://orcid.org/0000-0002-7847-1166
Centro Universitário São Lucas - UNISL/RO, Brasil
E-mail: cleberqueiroz05@hotmail.com

\begin{abstract}
Resumo
Os tumores estromais gastrointestinais (GISTs) são raros e representam 0,1 a $3 \%$ de todos os tumores gastrointestinais. $\mathrm{Na}$ maioria dos casos esses tumores se apresentam de forma assintomática, sendo descobertos incidentalmente por exames de imagem. A ecoendoscopia (EUS) tem sido utilizada como método de eleição para a caracterização destas lesões, permitindo avaliar com precisão o seu tamanho, camada de origem parietal e diversas características morfológicas. O diagnóstico é feito pela presença da proteína c-KIT (CD117) detectada pelo método imunohistoquímico. Os fatores prognósticos de grande importância são o tamanho do tumor e o índice mitótico. A ressecção cirúrgica é a terapia de escolha e o tratamento quimioterápico com mesilato de imatinibe está indicado nos casos de irressecabilidade ou doença metastática. Apresentamos um caso de GIST Duodenal diagnosticado pela ecoendoscopia após achado incidental durante exame de endoscopia digestiva alta.
\end{abstract}

Palavras-chave: Tumor estromal gastrointestinal; Proteína c-KIT; Trato gastrointestinal; Ecoendoscopia.

\begin{abstract}
Gastrointestinal stromal tumors (GISTs) are generally quite rare, but the same are the mesenchymal neoplasms that are most frequently identified in the gastrointestinal tract (GIT) and represent 0.1 to $3 \%$ of all gastrointestinal tumors. The diagnosis of this pathology is made according to the clinical picture, in the morphological characteristics, and fundamentally in the consideration of the presence of the protein c-KIT (CD117) detected by immunohistochemical method. In most cases, these tumors present in the individual asymptomatically, so they are discovered incidentally by imaging exams. The prognostic factors of great importance are the size of the tumor and the mitotic index. Surgical resection is the therapy of choice and imatinib mesylate chemotherapy, which is indicated in cases of unresectability or metastatic disease. We present a case of Duodenal GIST diagnoses by echoendoscopy after an incidental finding during an upper gastrointestinal endoscopy exam.
\end{abstract}

Keywords: Gastrointestinal stromal tumor; C-KIT Protein; Gastrointestinal tract; Endoscopy ultrasound.

\section{Resumen}

Los tumores del estroma gastrointestinal (GIST) son raros y representan del 0,1 al 3\% de todos los tumores gastrointestinales. En la mayoría de los casos, estos tumores se presentan de forma asintomática y se descubren de forma incidental mediante exámenes por imágenes. La ecoendoscopia (USE) se ha utilizado como método de elección para la caracterización de estas lesiones, permitiendo la valoración precisa de su tamaño, capa de origen parietal y diversas características morfológicas. El diagnóstico se realiza por la presencia de la proteína c-KIT (CD117) detectada por el método inmunohistoquímico. Los factores pronósticos de gran importancia son el tamaño del tumor y el índice mitótico. La resección quirúrgica es la terapia de elección y la quimioterapia con mesilato de imatinib está indicada en casos de irresecabilidad o enfermedad metastásica. Presentamos un caso de GIST duodenal diagnosticado por ecoendoscopia tras un hallazgo incidental durante la endoscopia digestiva alta.

Palabras clave: Tumor del estroma gastrointestinal; Proteína c-KIT; Tracto gastrointestinal; Ultrasonido. 


\section{Introdução}

Os tumores estromais gastrointestinais (GIST) caracterizam-se como tumores mesenquimais tendo uma frequência maior no trato digestivo (Lopasso, 2008). Eles preferencialmente se encontram no estômago (60\%), intestino delgado (25\%), colo e reto (10\%) e esôfago (5\%). A localização duodenal pode ocorrer, porém é considerada como raridade (3-5\% dos casos) (Dematteo et al., 2000).

O diagnóstico do GIST é feito pela presença de células fusiformes ou epitelioides na microscopia, com positividade na imuno-histoquímica para CD 117 ou C-Kit (95\% dos casos), e CD 34 (70\%) (Hirota et al., 1998). A ressecção cirúrgica com margens livres é o tratamento de escolha para os casos de GIST duodenal, sem que haja necessidade de esvaziamento linfonodal, já que raramente ocorre disseminação linfática nesta patologia (Garcia \& Blanco, 2009).

Os GISTs em grande parte dos casos são assintomáticos, assim são diagnosticados incidentalmente quando realizados análise através de imagem ou endoscópicos (Meesters et al., 1998). No início, o tumor geralmente não ocasiona sintomas, porém com seu crescimento geralmente surgem manifestações clínicas, que podem ser dor abdominal, sangramento, massa abdominal palpável, anemia ferropriva, sintomas dispépticos, emagrecimento e fenômenos obstrutivos (Yokoyama et al., 2007).

A avaliação do GIST é uma das indicações clássicas da Ecoendoscopia (EUS) (Mennigen et al., 2008). É a ferramenta mais importante para avaliar sua camada de origem, diagnóstico diferencial, classificação e acompanhamento dessas lesões (Beham et al., 2013).

Apresentamos um caso de um paciente com GIST duodenal diagnosticado pela ecoendoscopia com punção ecoguiada.

\section{Metodologia}

Os dados foram obtidos por meio da coleta de dados do prontuário, através da anamnese e exame físico da paciente concomitante aos exames de imagem. A paciente preencheu um formulário de consentimento, no momento da consulta, sobre seu quadro clínico, autorizando a utilização, de forma indireta, para fim científico. Para a revisão bibliográfica foram analisados artigos e periódicos que estavam relacionados com o tema.

\section{Relato de Caso}

Paciente feminina, 75 anos, natural da cidade do Rio de Janeiro-RJ, aposentada, procurou auxílio médico devido à presença de epigastralgia de forte intensidade. Portadora de hipertensão arterial sistêmica em uso regular de Perindopril e antecedente de cirurgia de adenoma de hipófise em 2018. Exame físico apresentando apenas dor leve à palpação profunda em epigástrio.

Realizada endoscopia digestiva alta que evidenciou esofagite de refluxo grau A de Los Angeles, hérnia de hiato por deslizamento, hiato alargado, gastrite endoscópica erosiva leve de Antro e uma lesão com aspecto subepitelial na segunda porção duodenal medindo cerca de 1,5 cm em seu maior eixo (Figura 1). As biópsias do estômago evidenciaram uma gastrite crônica leve com Helicobacter pylori, que foi tratado com esquema tríplice de erradicação.

Foi indicado a realização da ecoendoscopia que evidenciou uma lesão homogênea, hipoecóica, heterogênea, com bordos irregulares e pouco nítidos, apresentando na sua periferia diminutas imagens hiperecóicas com sombra acústica compatíveis com microcalcificações, originada na quarta camada ecogênica (muscular própria), medindo 1,0 x 1,0cm, distante 2,0 cm da Papila de Vater (Figura 2). Realizada punção ecoguiada da lesão com agulha de 22G - (fine needle aspiration - FNA) (Figura 3). A citologia mostrou agregado de células fusiformes sem sinais de malignidade e o estudo imuno-histoquímico para CD117 foi positivo caracterizando GIST (Figura 4).

Realizada nova endoscopia digestiva alta cerca de 6 meses após o primeiro exame diagnóstico e não houve alteração 
endoscópica em relação ao exame anterior.

Optou-se por manter acompanhamento clínico e endoscópico da paciente.

Figura 1. Lesão subepitelial na $2^{\mathrm{a}}$ porção duodenal.

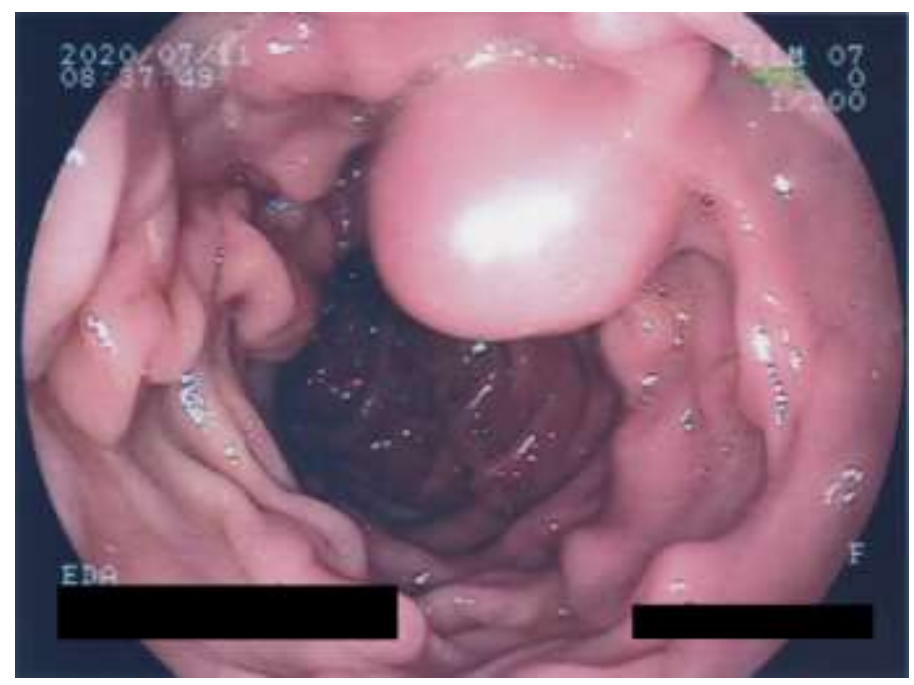

Fonte: Autores (2021).

Figura 2. Lesão hipoecóica com microcalcificações periféricas originada da muscular própria.

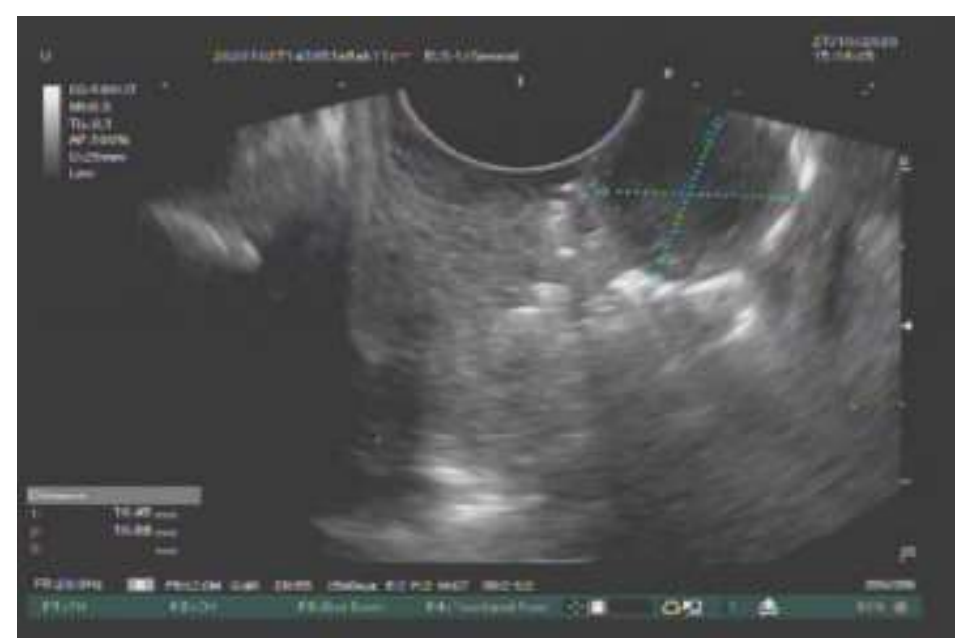

Fonte: Autores (2021). 
Figura 3. Agulha de punção ecoguiada no interior da lesão.

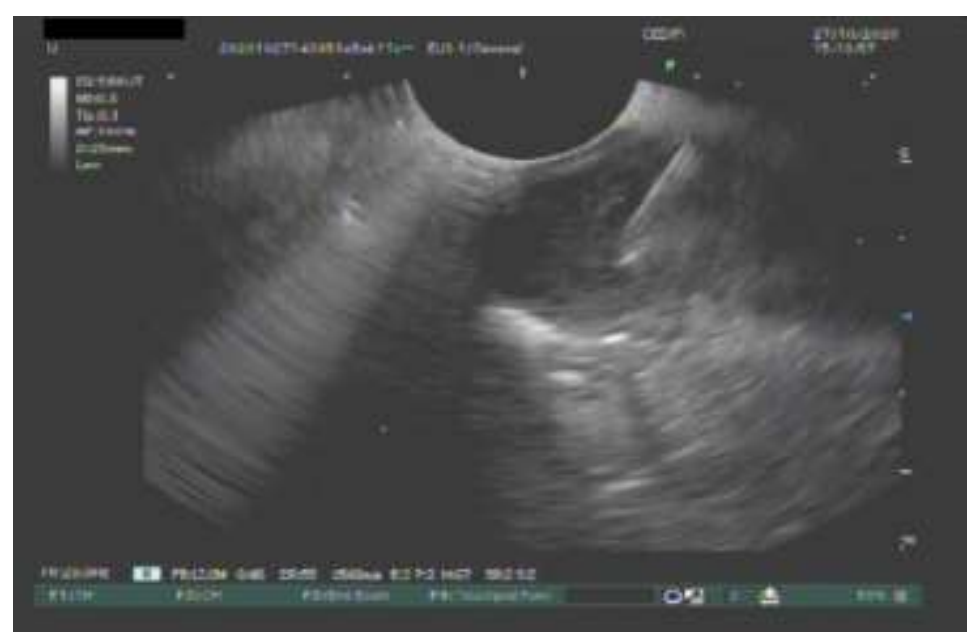

Fonte: Autores (2021).

Figura 4. Diagnóstico imuno-histoquímico de GIST.

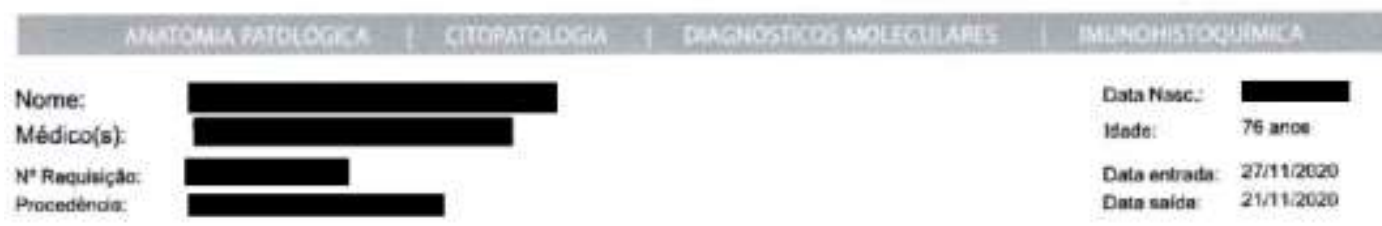

EXAME IMUNO-HISTOQUIMICO

\begin{tabular}{|c|c|c|c|}
\hline $\begin{array}{l}\text { Informaç̧óes clinicas } \\
\text { disponibillizadas }\end{array}$ & Lesîn subepitelal. & & \\
\hline \multicolumn{4}{|l|}{ Painel do Anticorpos } \\
\hline & Duodeno $z^{*}$ porceà & & \\
\hline & Antigeno & Anticorpe (clone) & Resullado \\
\hline & $\$ 100$ & Polchnal & negative \\
\hline & orsuiva & DE-R.11 & negetive \\
\hline & $\cos 4$ & OBEndio & negative. \\
\hline & $\operatorname{cot17}(\mathrm{km})$ & 0.7 & Positivo \\
\hline
\end{tabular}

Microscopia e parecer

diagnóstico

\section{Duodeno $Z^{*}$ porçî́: \\ PROLIFERACAO FUSOCELULAR FOCAL POSITINA PARA CD117 (KTT)}

Os achados indicam o diagnóetico de tumor estromal gastrointestinal (GIST). Camelacte com os demais aspectos clinico-endoscopicos è indicada para corroborar esse diagnóstica.

Fonte: Autores (2021).

\section{Discussão}

GIST são neoplasias raras que se originam das células de Cajal e expressam a proteína c-kit. Neste caso a imunohistoquímica mostrou proliferação fusocelular focal positiva para CD117 (cKIT) confirmando o diagnóstico de GIST (Uchida et al., 2015).

GIST são mais freqüentes no estômago (40\% a 70\% dos casos), seguido do intestino delgado e duodeno (20\% a $40 \%)$, e são raros no esôfago e no cólon (Consolo et al., 2007). O tratamento é cirúrgico, basicamente sem linfadenectomia e uma pequena margem de segurança de 1 a $2 \mathrm{~cm}$ é suficiente (Munõz et al., 2006). A doença residual pode ser tratada com o mesilato de Imatinib (Harris et al., 2020).

A Ecoendoscopia é o melhor exame para avaliação de lesões subepiteliais, como o GIST, podendo determinar tamanho, 
camada de origem, diagnóstico presuntivo, características ultrassonográficas malignas, realizar punção e orientar a conduta apropriada (Castro-Poças et al., 2015).

A conduta no GIST é bastante controversa (Hompes et al., 2004). Para GIST pequeno ( $<2 \mathrm{~cm})$, alguns estudos sugerem apenas acompanhamento, mas com o desenvolvimento de dispositivos e técnicas de modernas, a endoscopia vem ganhando espaço como alternativa de tratamento, como por exemplo as técnicas de tunelamento submucoso (STER) e de ressecção endoscópica (ESD, EFTR) (Chandrasekhara et al., 2107).

\section{Conclusão}

Em conclusão, apresentamos relato de caso de paciente com GIST duodenal de segunda porção $<1,0 \mathrm{~cm}$ diagnosticado pela ecoendoscopia com punção ecoguiada. O paciente está em acompanhamento clínico e endoscópico semestral, sem apresentar aumento da lesão.

\section{Referências}

Beham, A., Schaefer, I. M., Cameron, S., von Hammerstein, K., Füzesi, L., Ramadori, G., \& Ghadimi, M. B. (2013). Duodenal GIST: a single center experience. International journal of colorectal disease, 28(4), 581-590.

Casali, P. G., et al. (2009). Gastrointestinal stromal tumors: ESMO clinical recommendations for diagnosis, treatment and follow-up. Annals of Oncology, Oxford 20(1), 64-67.

Castro-Poças, F. M., Araújo, T. P., Silva, J. D., Lopes, C. A., \& Saraiva, M. M. (2015). Duodenal gastrointestinal stromal tumor and endoscopic ultrasound. Revista española de enfermedades digestivas, 107(12), 759-760.

Chandrasekhara, V., Khashab, M. A., Muthusamy, V. R., Acosta, R. D., Agrawal, D., Bruining, D. H., \& DeWitt, J. M. (2017). Adverse events associated with ERCP. Gastrointestinal endoscopy, 85(1), 32-47.

Consolo, F. D. S., Cardoso, A. A. P., Branco, O. M., Lamego, H., \& Arruda, L. (2007). Hemorragia digestiva por tumor estromal gástrico. Revista do Colégio Brasileiro de Cirurgiões, 34, 64-66.

D'amato, G., Steinert, D. M., McAuliffe, J. C., \& Trent, J. C. (2005). Update on the biology and therapy of gastrointestinal stromal tumors. Cancer control, 12(1), 44-56.

DeMatteo, R. P., Lewis, J. J., Leung, D., Mudan, S. S., Woodruff, J. M., \& Brennan, M. F. (2000). Two hundred gastrointestinal stromal tumors: recurrence patterns and prognostic factors for survival. Annals of surgery, 231(1), 51.

Garcia, A. Z. G., \& Blanco, A. P. (2009). Tumores del estroma gastrointestinal: tratamiento endoscópico. GH Continuada, Barcelona, 8(2). 76-81.

Harris, P. S., Romano, J., Russ, K. B., Shoreibah, M. G., \& Baig, K. R. K. K. (2020). Gastrointestinal Stromal Tumor: GIST Another Duodenal Ulcer. Ochsner Journal, 20(2), 236-238.

Hirota, S., Isozaki, K., Moriyama, Y., Hashimoto, K., Nishida, T., Ishiguro, S., \& Kitamura, Y. (1998). Gain-of-function mutations of c-kit in human gastrointestinal stromal tumors. Science, 279(5350), 577-580.

Hompes, D., Topal, B., Ectors, N., Aerts, R., \& Penninckx, F. (2004). Gastro-intestinal stromal tumour of the duodenum: extreme presentation in two cases. Acta Chirurgica Belgica, 104(1), 110-113.

Levy, A. D., Remotti, H. E., Thompson, W. M., Sobin, L. H., \& Miettinen, M. (2003). Gastrointestinal stromal tumors: radiologic features with pathologic correlation. Radiographics: a review publication of the Radiological Society of North America, Inc, 23(2), 283-304.

Lopasso, F. P. (2008). Neoplasias de estômago. In: Moraesfilho, J. P. P. Tratado das enfermidades gastrintestinais e pancreáticas. Roca, $722-724$.

Meesters, B., Pauwels, P. A. A., Pijnenburg, A. M., Vlasveld, L. T., \& van Driel, O. R. (1998). Metastasis in a 'benign'duodenal stromal tumour. European Journal of Surgical Oncology (EJSO), 24(4), 334-335.

Mennigen, R., Wolters, H. H., Schulte, B., \& Pelster, F. W. (2008). Segmental resection of the duodenum for gastrointestinal stromal tumor (GIST). World Journal of Surgical Oncology, 6(1), 1-6.

Mugayar, J. et al. (2008). Tumor estromal gastrointestinal. Conduta Médica, 9(36),10-15.

Muñoz, C., Sabah, S., Navarro, A., Planzer, M., Silva, C., \& Santander, R. (2006). Tumores del estroma gastrointestinal (GIST): Revisión de la literatura. Gastr Latinoam, 17(1), 43-51.

Rossini, L. G. B, Averbach, M.(2008). Endoscopia. In: MoraesFilho, Joaquim Prado Pinto. Tratado das enfermidades gastrintestinais e pancreáticas. 344. 
Research, Society and Development, v. 10, n. 11, e124101119407, 2021

(CC BY 4.0) | ISSN 2525-3409 | DOI: http://dx.doi.org/10.33448/rsd-v10i11.19407

Uchida, H., Sasaki, A., Iwaki, K., Tominaga, M., Yada, K., Iwashita, Y., \& Kitano, S. (2005). An extramural gastrointestinal stromal tumor of the duodenum mimicking a pancreatic head tumor. Journal of hepato-biliary-pancreatic surgery, 12(4), 324-327.

Yokoyama, R. M. et al. Tumor estromal gastrointestinal. Revista Brasileira de Videocirurgia, 5(2), 65-71. 